\title{
Variable Neighborhood Search based algorithms for high school timetabling
}

\author{
George H.G. Fonseca ${ }^{a}$, Haroldo G. Santos ${ }^{b}$ \\ ${ }^{a}$ Computing and Systems Department, Federal University of Ouro Preto, Brazil \\ ${ }^{\mathrm{b}}$ Computing Department, Federal University of Ouro Preto, Brazil
}

\section{A R T I C L E I N F O}

Available online 6 February 2014

Keywords:

Variable Neighborhood Search

High School Timetabling Problem

Third International Timetabling

Competition

\begin{abstract}
A B S T R A C T
This work presents the application of Variable Neighborhood Search (VNS) based algorithms to the High School Timetabling Problem. The addressed model of the problem was proposed by the Third International Timetabling Competition (ITC 2011), which released many instances from educational institutions around the world and attracted 17 competitors. Some of the VNS algorithm variants were able to outperform the winner of Third ITC solver, which proposed a Simulated Annealing - Iterated local Search approach. This result coupled with another reports in the literature points that VNS based algorithms are a practical solution method for providing high quality solutions for some hard timetabling problems. Moreover they are easy to implement with few parameters to adjust.
\end{abstract}

(c) 2014 Published by Elsevier Ltd.

\section{Introduction}

The High School Timetabling Problem is faced by many educational institutions around the world. The basic search version consists in assigning teacher $\times$ class activities to timeslots and rooms in such a way that no teacher, class or room is involved with more than one event at time. Generally, this assignment is repeated weekly until the end of the semester. Many other constraints are considered in real problems, like availability of teachers, to avoid idle times and to limit the number of lessons of the same subject taught to a class in a day.

Beyond its practical importance, this problem was proven to be $\mathcal{N P}$-Hard [1,2]. Progress in heuristic and exact approaches for tackling these problems is a major goal of current research in Operations Research and Artificial Intelligence.

Three international competitions (ITCs) were made to bring the attention of scientists and practitioners for this problem, with the objective of performing comparisons of different methods in a controlled computational environment: the first one happened in 2003 [3] and was won by Kostuch [4] with a 3-phase Simulated Annealing (SA) [5] based approach. In 2007, the second one [6] started and was composed of three separated tracks, which were mostly won by Müller [7] also with a Simulated Annealing based approach. The last one [8] happened in 2012 and was won by a Simulated Annealing - Iterated Local Search [9] approach.

As the results of the competitions show, local search methods are defining the state-of-art heuristic solvers for educational

E-mail addresses: george@decsi.ufop.br (G.H.G. Fonseca), haroldo@iceb.ufop.br (H.G. Santos). timetabling problems. Specially, the Simulated Annealing metaheuristic composed the solver of all winners. The role of exact methods which employ Integer Programming, such as the proposed in [10-12], appears to be still very limited for tackling the problems and instances which appeared in these competitions, considering the absence of these techniques in submissions. This scenario contrasts with the first International Nurse Rostering Competition [13], for instance, where two of the first places used Integer Programming in some form.

This paper presents a computational study of Variable Neighborhood Search and its variants applied to the Third ITC problem. The results indicate that the proposed method outperforms the state-of-art method.

The remaining of this work is organized as follows: Section 2 presents the problem considered in this paper, the Third ITC problem; Section 3 presents our solution approach; Section 4 presents computational experiments and finally, Section 5 concludes our paper and discusses future works.

\section{High School Timetabling Problem model}

The roots of the School Timetabling model considered in this paper, the model of the Third ITC, are in the Benchmarking project for (High) School Timetabling. ${ }^{1}$ The project, which involved a group of researchers in this area, started with the ambitious goal of developing a XML format capable of modeling different school

\footnotetext{
${ }^{1}$ http://www.utwente.nl/ctit/hstt/
} 
timetabling problems arising in diverse institutions around the world. Initial versions of this project appeared in the PATAT 2008 conference [14], with an improved version named XHSTT published later [15]. Nowadays, the project site holds approximately 50 datasets from 11 countries. The project site also includes an evaluator to validate solutions and the best known solutions are kept, so that the results of newly proposed methods can be immediately confronted with previously obtained results. Some of the previous models which are now in XHSTT are [16-20,10,21]. The model is split into three main entities: Time and Resources, Events and Constraints. A solution consists of a set of assignments of times and resources to the events.

\subsection{Times and resources}

The time entity consists of a timeslot, which is an indivisible interval of time. Timeslots do not overlap and can be grouped in timegroups. Resources are entities which attend events. Typical resources are students, teachers and rooms [21]:

Students: a group of students attends events (lessons); important constraints associated with students are the control of their idle times and the number of lessons taken by day.

Teachers: teachers perform their academic tasks in events; the allocation of teachers for specific teaching activities can be preassigned or not; when teachers are not preassigned, they should be assigned according to their qualifications and workload limits.

Rooms: the usage of rooms for hosting events must be observed: some events require rooms with a given capacity and/or a set of special features.

\subsection{Events}

An event (instance event) is a meeting between resources, usually representing a simple lesson or a set of lessons (event group). Each instance event needs to be scheduled into one or more solution events. Timeslot assignments to events are called meets and the assignment of resources to events is tasks. The term course is used to designate a group of students who attend to the same events. Other kinds of events, like meetings, are allowed by the model [21]. The following attributes can be specified for events, the first one is the only obligatory:

Duration: represents the number of timeslots which have to be assigned to the event.

Course: a course is a grouping of events: events declared in the same course constitute a course of study in one subject for one group of students.

Pre-assigned resources: to attend the event.

Workload: that will be added to the total workload of resources assigned to the event.

Pre-assigned timeslot: some events have only one timeslot in which they can be assigned.

\subsection{Constraints}

Post et al. [21] group the constraints into three categories: basic constraints of scheduling, constraints of events and constraints of resources. The objective function $f(\cdot)$ is computed considering the summation of penalties for deviations in different constraints and events/resources which they refer. The flexibility of XHSTT allows the inclusion of non-linear terms in the cost function which is used to compute the penalties [15]. The constraints are also divided into hard constraints, whose satisfaction is mandatory; and soft constraints, whose satisfaction is desirable but not obligatory. Costs for violations in these two types of constraints are summed in two separated costs: the infeasibility cost and the quality cost, defining a hierarchical objective function. Each instance can define whether a constraint is hard or soft, its weight and the type of cost function used (eg. linear or quadratic). For more details, see [15].

\subsubsection{Basic constraints of scheduling}

1. Assign Time: assign timeslots to each event.

2. Assign Resource: assign the resources to each event.

3. Prefer Times: indicates that some event have preference for a particular timeslot(s).

4. Prefer Resources: indicates that some event have preference for a particular resource(s).

\subsubsection{Constraints to events}

1. LINK EVENTS: to schedule a set of events to the same starting time.

2. SPREAD Events: specify the allowed number of occurrences for event groups in time groups between a minimum a maximum number of times; this constraint can be used, for example, to define a daily limit of lessons.

3. Avoid Split Assignments: for each event, assign a particular resource to all of its meets.

4. Distribute Split Events: for each event, assign between a minimum and a maximum meets of a given duration.

5. Split Events: limits the number of non-consecutive meets that an event should be scheduled and its duration.

\subsubsection{Constraints to resources}

1. Avoid Clashes: assign the resources without clashes (i.e. without assign the same resource to more than one event at a timeslot).

2. Avoid Unavailable Times: avoid assigning resources on the times that they are not available.

3. LIMIT WORKLOAD CONSTRAINT: schedule the workload of the resources between a minimum and a maximum bound.

4. Limit Idle Times: the number of idle times in each time group should lie between a minimum and a maximum bound for each resource; typically, a time group consists of all timeslots of a given week day.

5. Limit Busy Times: the number of busy times in each time group should lie between a minimum and a maximum bound for each resource.

6. Cluster Busy Times: the number of time groups with a timeslot assigned to a resource should lie between a minimum and a maximum limit; this can be used, for example, to concentrate teacher's activities in as few days as possible.

\section{Solution approach}

Our approach uses the Kingston High School Timetabling Engine (KHE) [22] to generate initial solutions. Afterwards, we implemented the Variable Neighborhood Search metaheuristic and some of its variants to perform local search around this solution. These elements will be explained in the following subsections.

\subsection{Build method}

The KHE is a platform for handling instances of the addressed problem. It also provides a solver, used to build initial solutions in 
the presented approach. This solver was chosen to generate the initial solutions since it is able to find reasonably good initial solutions in short amounts of time.

The incorporated solver is based on the concept of Hierarchical Timetabling [23], where smaller allocations are joined to generate bigger blocks of allocation until a full representation of the solution is developed. Hierarchical Timetabling is supported by the Layer Tree data structure [23], consisting of nodes that represent the required meet and task allocation. An allocation may appear in at most one node. A Layer is a subset of nodes having the propriety that none of them can be overlapped in time. Commonly, nodes are grouped in a Layer when share resources.

The hard constraints of the problem are modeled to this data structure and then a Matching problem is solved to find the times/ resources allocation. The Matching is done by connecting each node to a timeslot or resource respecting the property of Layer. For full details, see $[23,22]$.

\subsection{Neighborhood structure}

Six neighborhood structures were used:

1. Event Swap (ES): Two events $e_{1}$ and $e_{2}$ have their timeslots $t_{1}$ and $t_{2}$ swapped respectively.

2. Event Move (EM): An event $e_{1}$ is moved from timeslot $t_{1}$ to another timeslot $t_{2}$.

3. Event Block Move (Евм): Works like Es, but when moving events with different durations in contiguous timeslots, keeps these events adjacent.

4. Resource Swap (RS): Two events $e_{1}$ and $e_{2}$ have their assigned resources $r_{1}$ and $r_{2}$ swapped respectively. Resources $r_{1}$ and $r_{2}$ should play the same role to allow the swap (e.g. both have to be teachers).

5. Resource Move (RM): An event $e_{1}$ has its assigned resource $r_{1}$ replaced by a new resource $r_{2}$.

6. Kempe Move (Км): Two times $t_{1}$ and $t_{2}$ are fixed and one seeks the best path at the bipartite conflict graph containing all events in $t_{1}$ and $t_{2}$; arcs are built from conflicting events which are in different timeslots and their cost is the cost of swapping the timeslots of these two events.

The set of neighborhoods is quite similar to the one used in Fonseca [9].

\subsection{Variable Neighborhood Search}

The Variable Neighborhood Search Method was proposed by Mladenovic and Hansen [24] and consists in a local search method that explores the search space by making systematic changes in the neighborhood structures.

In each iteration, a neighborhood structure $k$ is selected according to the order presented in Section 3.2. A random neighbor $s^{\prime}$ is generated in this neighborhood. Afterwards, a descent method is applied to $s^{\prime}$. If the best solution found by descent method, $s^{\prime \prime}$, is better than the best known solution, it is updated and the neighborhood structure is set to the first one. Otherwise, the search continues in the next neighborhood structure. When we explore the last neighborhood structure $k_{\max }=6$, the search goes back to the first neighborhood. This process continues until a stop condition is reached.

A key component of VNS algorithms is the descent phase (Algorithm 1, line 5). The ability to quickly reach good local optima is critical to the success of the method. Our implementation aims at the fast generation of high quality solutions which tend to be local optima with respect to many neighborhoods. Thus, at each iteration of the descent phase, a different neighborhood can be considered, with the following probabilities of selection: if the instance requires the assignment of resources (i.e. there exists at least one AsSIGN RESOURCE constraint), the neighborhood is chosen based on the following probabilities: $\mathrm{ES}=0.20, \mathrm{EM}=0.38, \mathrm{EBM}=0.10, \mathrm{RS}=0.20, \mathrm{RM}=0.10$ and $\mathrm{KM}=0.02$. Otherwise, the neighborhoods Rs and RM are not used and the odds become $\mathrm{ES}=0.40, \mathrm{EM}=0.38, \mathrm{EBS}=0.20$ and $\mathrm{KM}=0.02$. Since the union of all these neighborhoods is usually a very large search space, composed of many flat landscapes, we employed Random NonAscendent (RNA) movements in the descent phase, with the stopping criterion of 1,000,000 non-improvement iterations. These values were empirically adjusted.

Algorithm 1 presents the basic implementation of VNS, denoted here as BVNS. Note that the adopted stop condition is a timeout, to be discussed in Section 4. Some variations of VNS implemented are present in the following subsections. Some successful examples of application of VNS can also be found in [25-27].

Algorithm 1. Basic VNS (BVNS) algorithm.

Input: Initial solution $s$.

Output: Best solution $s$ found.

1 while elapsedTime $<$ timeout do

$2 \mid k \leftarrow 1$;

3 while $k \leq k_{\max }$ do

$4 \quad \mid$ Generate a random neighbor $s^{\prime} \in N_{k}(s)$;

$5 \quad s^{\prime \prime} \leftarrow$ descentMethod $\left(s^{\prime}\right)$;

6 if $f\left(s^{\prime \prime}\right) \leq f(s)$ then

$7 \quad \mid s \leftarrow s^{\prime \prime}$;

$8 \quad \quad k \leftarrow 1$;

9 else

10

$k \leftarrow k+1$

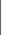

11 return $s$;

\subsubsection{Reduced Variable Neighborhood Search}

A reduction to the original Variable Neighborhood Search Method was also proposed by Mladenovic and Hansen [24] in which we do not have a descent phase (Algorithm 1, line 5) to improve the generated solution $s^{\prime}$ at each iteration. This may improve the VNS performance in cases in which the complete exploration of the defined neighborhoods is too computationally expensive. This reduction was called Reduced Variable Neighborhood Search (RVNS).

\subsubsection{Sequential Variable Neighborhood Descent}

Another variation of the original VNS method is the Sequential Variable Neighborhood Descent (SVND) [28]. The main difference between the basic VNS and SVND method is instead of allowing all neighborhood structures to be explored in the descent phase, we allow only a subset of the available neighborhood structures at each iteration. In our implementation, we made the local search at each iteration considering only one neighborhood structure $k$ $\left(s^{\prime \prime} \leftarrow \operatorname{descentMethod}_{k}\left(s^{\prime}\right)\right)$.

\subsubsection{Skewed Variable Neighborhood Search}

Taking larger and larger neighborhoods, the information related to the best local optimum dissolves and VNS degenerates into multistart [29]. To deal with these cases a new variant of VNS, the Skewed Variable Neighborhood Search (SVNS), was proposed. In this variant, we have a relaxed rule to accept the candidate solution $s^{\prime \prime}$. The relaxed rule uses an evaluation function linear in the distance from the incumbent: $f\left(s^{\prime \prime}\right)$ is replaced by $f\left(s^{\prime \prime}\right)-\alpha$ $\times \rho\left(s, s^{\prime \prime}\right)$, where $\rho\left(s, s^{\prime \prime}\right)$ is the distance from $s$ to $s^{\prime \prime}$ and $\alpha$ a 
parameter. To compute the distance between two solutions we used the following metric: for each solution we compute a string with $n$ positions, where $n$ is the number of events. In each position there is an ordered pair indicating the meeting and tasks which are associated with this event. Then, $\rho\left(s, s^{\prime \prime}\right)$ is the Hamming distance of these two strings. After some experiments, we set $\alpha=1.0$.

In our implementation, we made the local search at each iteration considering only one neighborhood structure $k\left(s^{\prime \prime} \leftarrow\right.$ descentMethod $_{k}\left(s^{\prime}\right)$ ).

\section{Computational experiments}

All experiments ran on an Intel ${ }^{\circledR}$ Core i5 $2.4 \mathrm{GHz}$ computer with 4 GB of RAM under the Ubuntu 11.10 operating system. The programming language used was $\mathrm{C}++$ compiled with the GNU Compiler Collection version 4.6.1. All generated solutions were validated by the HSEval validator (http://sydney.edu.au/engineer ing/it/ jeff/hseval.cgi). We considered the timeout of the competition in all experiments, which was $1000 \mathrm{s.}^{2}$

Results are expressed by the pairs $x / y$, where $x$ contains the feasibility measure and $y$ the quality measure. Our solver along with our solutions and reports can be found in https://sites.google. $\mathrm{com} /$ site/georgehgfonseca/producaoacademica/vns.rar. We invite the interested reader to validate our results.

\subsection{Dataset characterization}

The set of instances available from Third ITC http://www. utwente.nl/ctit/hstt/archives/XHSTT-2012 was originated from many countries and ranges from small instances to huge challenging ones. Table 1 presents the main features of these problems.

\subsection{Obtained results}

In the first set of experiments we evaluated the proposed methods using the same metric employed in the Third ITC : average results produced in a restricted time limit, as discussed in the beginning of this section. Table 2 presents the obtained average results of the basic VNS method (BVNS) and its variations: RVNS, SVND and SVNS. We also included in this table the results of the KHE engine [30] initial solutions as well as the results of the Third ITC winner, a Simulated Annealing-Iterated Local Search approach [9]. These results are presented in columns KHE and SAILS. Each cell includes the average result of five independent executions ${ }^{3}$ of one method on a given instance.

Table 3 presents the ordering of the presented methods according to the Third ITC rules. Each solver receives a rank in each instance ranging from 1 (best) to 4 (worst) according to the average costs of solutions obtained. The solver with the smaller average rank is considered the best.

Brito et al. [31] presented another VNS based approach to this problem. In their work, they used the Simulated Annealing algorithm to perform the local search at each iteration of VNS. They used the public set of instances from ITC to evaluate their approach since the hidden set was not released yet.

Table 4 presents the comparison between the SVNS method and the results presented by Brito et al. [31]. The best result on each instance is highlighted in bold.

\footnotetext{
${ }^{2}$ The CPU time was adjusted in our computer using the ITC benchmark software, which suggested $1500 \mathrm{~s}$.

${ }^{3}$ Random seeds from 1 to 5 .
}

Table 1

Features of considered instances from Third ITC.

\begin{tabular}{llllrc}
\hline Instance & Times & Teachers & Rooms & Classes & Lessons \\
\hline BrazilInstance2 & 25 & 14 & & 6 & 150 \\
BrazilInstance3 & 25 & 16 & & 8 & 200 \\
BrazilInstance4 & 25 & 23 & & 12 & 300 \\
BrazilInstance6 & 25 & 30 & & 14 & 350 \\
FinlandElementarySchool & 35 & 22 & 21 & 291 & 445 \\
FinlandSecondarySchool2 & 40 & 22 & 21 & 469 & 566 \\
Aigio1stHighSchool10-11 & 35 & 37 & & 208 & 532 \\
Italy_Instance4 & 36 & 61 & & 38 & 1101 \\
KosovaInstance1 & 62 & 101 & & 63 & 1912 \\
Kottenpark2003 & 38 & 75 & 41 & 18 & 1203 \\
Kottenpark2005A & 37 & 78 & 42 & 26 & 1272 \\
Kottenpark2008 & 40 & 81 & 11 & 34 & 1118 \\
Kottenpark2009 & 38 & 93 & 53 & 48 & 1301 \\
Woodlands2009 & 42 & 40 & & & 1353 \\
Spanishschool & 35 & 66 & 4 & 21 & 439 \\
WesternGreeceUniversity3 & 35 & 19 & & 6 & 210 \\
WesternGreeceUniversity4 & 35 & 19 & & 12 & 262 \\
WesternGreeceUniversity5 & 35 & 18 & & 6 & 184 \\
\hline
\end{tabular}

\subsection{Discussion of results}

For some instances, even the production of feasible solutions configures a hard task. These instances commonly define most constraints as hard constraints. The VNS approach and its variations were able to find 12 out of 18 feasible solutions to the instance set, one more than the Third ITC winner.

As it can be seen in Table 2, the VNS based approach was able to outperform the Third ITC winner. More specifically, the SVNS algorithm presented better results in most of the instances. One explanation to this result may be the fact that SVNS has an improved mechanism to escape from large valleys which does not rely only on randomness.

To understand the positive effect of the controlled diversification in SVNS we plotted in Fig. 1 the evolution in time of the relative distance (gap) of the cost of the incumbent solution to the best known solution in BVNS and SVNS for two hard instances. Considering the incumbent solution cost $c$ and the best known solution cost $c^{*}$ the gap is computed at each time instant as $\left(c-c^{*}\right) / c^{*}$. The cost is a fixed point value where the integer part corresponds to the feasibility cost and the fractional part to the quality cost. As it can be seen in Fig. 1, while in the beginning of the search both methods are comparable, SVNS improves solutions much more often as the search process advances.

The algorithm RVNS presented a poor performance. We believe that the fact that it does not systematically reach different local optima contributed to these poor results. Moreover, an excessive exploration in larger, more expensive neighborhoods, may also slow down the search in cases where improvement movements could be found in smaller neighborhoods.

We compared the method which found the best results, SVNS, to the SA-VNS approach presented by Brito et al. [31]. SVNS was able to outperform the SA-VNS results in 14 out of 19 instances. This result points that a descent method may be more effective than the Simulated Annealing method to perform local search at each iteration of VNS for this problem. The RNA descent method which we implemented has a smaller computational cost and has only one parameter to tune. Note that SA-VNS performed better than SVNS in the easy instances, since the computational cost of Simulated Annealing is not a problem in these cases.

\section{Concluding remarks}

The VNS algorithm showed strong results when applied to the High School Timetabling Problem, outperforming the Third ITC 
Table 2

Average results produced with VNS variants and other approaches in the restricted time limit of the Third ITC.

\begin{tabular}{|c|c|c|c|c|c|c|}
\hline Instance & KHE [30] & SA-ILS [9] & BVNS & RVNS & SVND & SVNS \\
\hline BrazilInstance2 & $4 / 90$ & $1.0 / 63.9$ & $0.0 / 40.6$ & $2.2 / 71.4$ & $0.6 / 63.8$ & $0.0 / 39.6$ \\
\hline BrazilInstance3 & $3 / 240$ & $0.0 / 127.8$ & $0.0 / 113.0$ & $2.4 / 151.4$ & $1.6 / 136.8$ & $0.0 / 119.0$ \\
\hline BrazilInstance4 & $39 / 144$ & 17.2/99.6 & $4.8 / 108.2$ & $21.0 / 112.8$ & $13.6 / 103.4$ & $3.8 / 123.4$ \\
\hline BrazilInstance6 & $11 / 291$ & $4.0 / 223.5$ & $0.0 / 157.4$ & $6.0 / 271.0$ & $2.2 / 231.2$ & $0.0 / 151.4$ \\
\hline FinlandElementarySchool & $9 / 30$ & $0.0 / 4.0$ & $0.0 / 3.4$ & $2.6 / 7.4$ & $0.0 / 4.0$ & $0.0 / 3.8$ \\
\hline FinlandSecondarySchool2 & $2 / 1821$ & $0.0 / 0.4$ & $0.0 / 0.4$ & $0.6 / 86.8$ & $0.0 / 1.0$ & $0.0 / 0.4$ \\
\hline Aigio1stHighSchool10-11 & $14 / 757$ & $0.0 / 15.3$ & $0.4 / 10.2$ & $11.2 / 200.0$ & $4.8 / 259.0$ & $0.2 / 8.2$ \\
\hline ItalyInstance 4 & $39 / 21,238$ & $0.0 / 658.4$ & $0.0 / 409.0$ & $0.4 / 2666.6$ & $0.0 / 1271.0$ & $0.0 / 324.8$ \\
\hline Kosovalnstance 1 & $1333 / 566$ & $14.0 / 6934.4$ & $1.2 / 20.4$ & $31.6 / 278.8$ & $2.0 / 75.6$ & $1.2 / 17.4$ \\
\hline Kottenpark2003 & $3 / 78,440$ & $0.6 / 90,195.8$ & $2.0 / 10,217.2$ & $2.4 / 34,766.0$ & $2.8 / 7937.8$ & $2.0 / 9694.4$ \\
\hline Kottenpark2005A & $35 / 23,677$ & $33.9 / 27,480.4$ & $33.8 / 19,059.2$ & $35.0 / 22,914.0$ & $27.0 / 10,118.0$ & $33.8 / 18,547.6$ \\
\hline Kottenpark2008 & $63 / 140,083$ & $25.7 / 31,403.7$ & $15.6 / 23,962.0$ & $36.8 / 38,936.6$ & $16.8 / 33,443.6$ & $15.8 / 24,024.2$ \\
\hline Kottenpark2009 & $55 / 211,095$ & $36.6 / 15,4998.5$ & $35.0 / 8543.0$ & $45.4 / 148,601.0$ & $31.2 / 8563.0$ & $33.2 / 9667.0$ \\
\hline Woodlands2009 & $19 / 0$ & $2.0 / 15.8$ & $2.0 / 8.2$ & $10.8 / 16.4$ & $2.0 / 14.4$ & $2.0 / 6.2$ \\
\hline Spanish school & $1 / 4103$ & $0.0 / 865.2$ & $0.0 / 907.8$ & $0.0 / 3068.0$ & $0.0 / 1126.0$ & $0.0 / 724.2$ \\
\hline WesternGreeceUniversity3 & $0 / 30$ & $0.0 / 5.6$ & $0.0 / 5.4$ & $0.0 / 20.4$ & $0.0 / 15.2$ & $0.0 / 5.0$ \\
\hline WesternGreeceUniversity4 & $0 / 41$ & $0.0 / 7.4$ & $0.0 / 6.4$ & $0.0 / 30.0$ & $0.0 / 23.6$ & $0.0 / 5.6$ \\
\hline WesternGreeceUniversity5 & $17 / 44$ & $0.0 / 0.0$ & $0.0 / 0.0$ & $2.8 / 16.2$ & $1.2 / 3.0$ & $0.0 / 0.0$ \\
\hline Average & $91.5 / 26,816.11$ & $7.5 / 17,394.4$ & $5.3 / 3531.8$ & 11.7/14,011.9 & $5.9 / 3521.7$ & $5.1 / 3525.7$ \\
\hline
\end{tabular}

Table 3

Solvers ranking.

\begin{tabular}{llllll}
\hline Instance & SA-ILS [9] & BVNS & RVNS & SVND & SVNS \\
\hline BrazilInstance2 & 4.0 & 2.0 & 5.0 & 3.0 & 1.0 \\
BrazilInstance3 & 3.0 & 1.0 & 5.0 & 4.0 & 2.0 \\
BrazilInstance4 & 4.0 & 2.0 & 5.0 & 3.0 & 1.0 \\
BrazilInstance6 & 4.0 & 2.0 & 5.0 & 3.0 & 1.0 \\
FinlandElementarySchool & 3.5 & 1.0 & 5.0 & 3.5 & 2.0 \\
FinlandSecondarySchool2 & 2.0 & 2.0 & 5.0 & 4.0 & 2.0 \\
Aigio 1st High School 2010-2011 & 1.0 & 3.0 & 5.0 & 4.0 & 2.0 \\
Italy_Instance4 & 3.0 & 2.0 & 5.0 & 4.0 & 1.0 \\
KosovaInstance1 & 4.0 & 2.0 & 5.0 & 3.0 & 1.0 \\
Kottenpark2003 & 1.0 & 3.0 & 4.0 & 5.0 & 2.0 \\
Kottenpark2005A & 4.0 & 3.0 & 5.0 & 1.0 & 2.0 \\
Kottenpark2008 & 4.0 & 1.0 & 5.0 & 3.0 & 2.0 \\
Kottenpark2009 & 4.0 & 3.0 & 5.0 & 1.0 & 2.0 \\
Woodlands2009 & 4.0 & 2.0 & 5.0 & 3.0 & 1.0 \\
Spanish school & 2.0 & 3.0 & 5.0 & 4.0 & 1.0 \\
WesternGreeceUniversityInstance3 & 3.0 & 2.0 & 5.0 & 4.0 & 1.0 \\
WesternGreeceUniversityInstance4 & 3.0 & 2.0 & 5.0 & 4.0 & 1.0 \\
WesternGreeceUniversityInstance5 & 2.0 & 5.0 & 4.0 & 2.0 & 2.0 \\
Average & 3.08 & 2.28 & 4.89 & 3.25 & 1.50 \\
\hline
\end{tabular}

Table 4

Comparative between SA-VNS approach [31] and SVNS approach.

\begin{tabular}{lrrr}
\hline Instance & SA-VNS $[31]$ & SA-RVNS $[31]$ & \multicolumn{1}{c}{ SVNS } \\
\hline AustraliaBGHS98 & $11 / 475$ & $11 / 475$ & $\mathbf{9 / 4 1 1}$ \\
AustraliaSAHS96 & $\mathbf{1 8} / \mathbf{5 2}$ & $18 / 52$ & $19 / 30$ \\
AustraliaTES99 & $9 / 187$ & $9 / 187$ & $\mathbf{9 / 1 7 7}$ \\
Brazillnstance1 & $0 / 21$ & $0 / 44$ & $\mathbf{0} / \mathbf{1 7}$ \\
BrazilInstance4 & $12 / 123$ & $12 / 153$ & $\mathbf{1 / 9 0}$ \\
BrazilInstance5 & $4 / 148$ & $4 / 184$ & $\mathbf{0} / \mathbf{7 8}$ \\
BrazilInstance6 & $4 / 213$ & $4 / 213$ & $\mathbf{0} / \mathbf{1 5 1}$ \\
BrazilInstance7 & $11 / 267$ & $11 / 318$ & $\mathbf{0 / 2 4 2}$ \\
EnglandStPaul & $2 / 48,758$ & $2 / 48,450$ & $\mathbf{1 / 2 6 , 2 5 8}$ \\
FinlandArtificialSchool & $19 / 12$ & $19 / 12$ & $\mathbf{6 / 5}$ \\
FinlandCollege & $\mathbf{1 / 4 9}$ & $1 / 77$ & $2 / 32$ \\
FinlandHighSchool & $\mathbf{0 / 1 6}$ & $0 / 73$ & $0 / 29$ \\
FinlandSecondarySchool & $\mathbf{0 / 1 1 4}$ & $0 / 129$ & $1 / 94$ \\
GreecePatras3rdHS2010 & $0 / 12$ & $0 / 20$ & $\mathbf{0 / 0}$ \\
GreecePreveza3rdHS2008 & $0 / 37$ & $0 / 33$ & $\mathbf{0} / \mathbf{4}$ \\
ItalyInstance1 & $\mathbf{0 / 2 0}$ & $0 / 31$ & $0 / 24$ \\
Kottenpark2003 & $1 / 72,413$ & $0 / 85,372$ & $\mathbf{0 / 9 3 6 5}$ \\
Kottenpark2005 & $20 / 28,710$ & $20 / 28,482$ & $\mathbf{1 8 / 1 0 , 0 5 2}$ \\
SouthAfricaLewitt2009 & $0 / 78$ & $0 / 74$ & $\mathbf{0} / \mathbf{8}$ \\
\hline
\end{tabular}
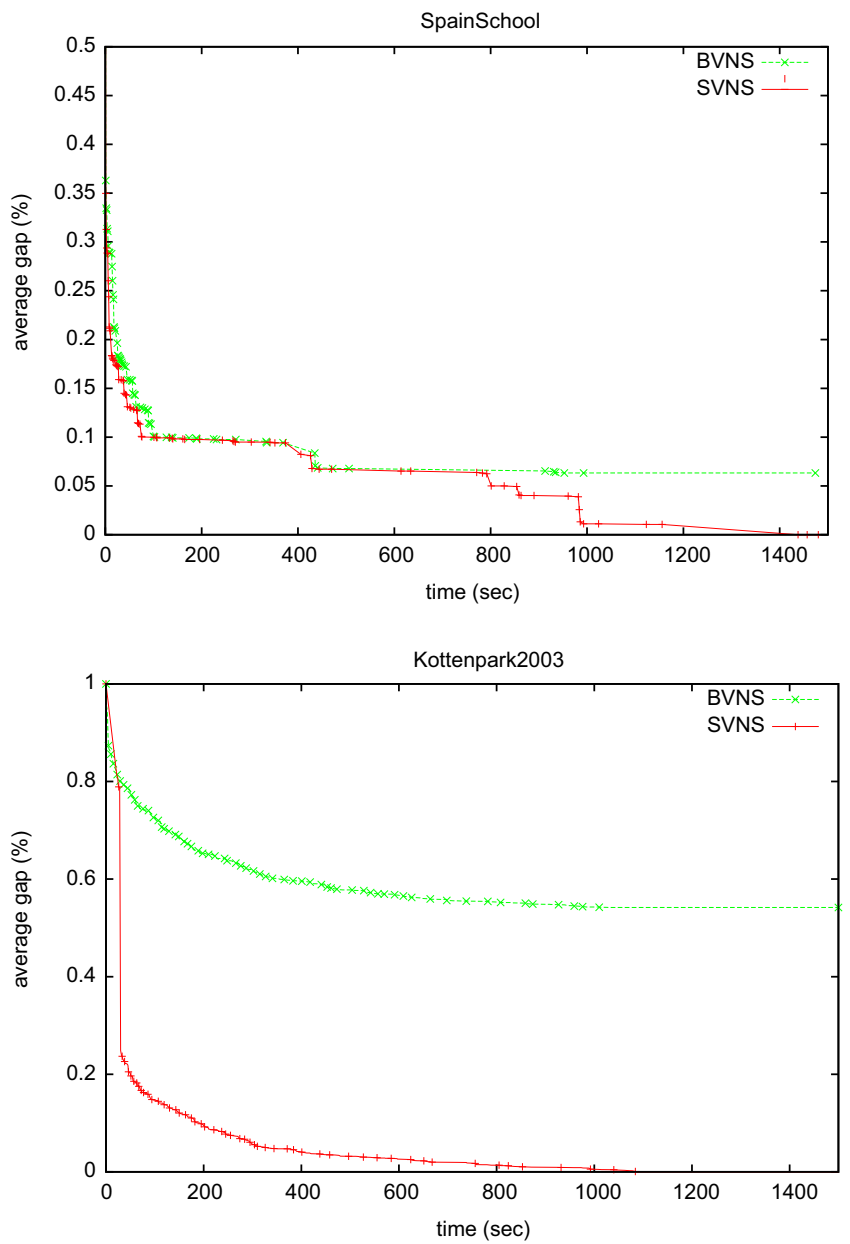

Fig. 1. Evolution of the distance to the best known solution in the search process of BVNS and SVNS.

winner approach. This result coupled with another recent reports in literature [25-27,32] points that VNS and its variations are very good alternatives for the heuristic solution of timetabling and scheduling problems.

Contrary to the tradition established by the last three timetabling competitions, where the best timetabling solvers incorporated 
Simulated Annealing in some form, we demonstrated that a proper implementation of the Skewed VNS method provides an excellent heuristic for the High School Timetabling Problem. We consider that the VNS approach has two important advantages when compared to SA based approaches: VNS usually has less parameters to tune and these parameters are not sensible to scales.

Some possible future works are to (1) implement and evaluate another metaheuristics to this problem, like evolutionary algorithms; (2) implement other neighborhood movements; and (3) develop a graphical user interface and allow schools and universities from all around the world to produce their instances and solve them with our solver.

\section{References}

[1] Even S, Itai A, Shamir A. On the complexity of timetable and multicommodity flow problems. SIAM J Comput 1976;5(4):691-703.

[2] Garey MR, Jonhson DS. Computers and intractability: a guide to the theory of NP-completeness. San Francisco, CA, USA: Freeman; 1979.

[3] IDSIA. International Timetabling Competition 2002. Available at $\langle$ http://www. idsia.ch/Files/ttcomp2002//; 2012 [accessed December 2012].

[4] Kostuch P. The university course timetabling problem with a three-phase approach. In: Proceedings of the 5th international conference on practice and theory of automated timetabling. Berlin, Heidelberg: Springer-Verlag; 2005. p. 109-25.

[5] Kirkpatrick S, Gellat DC, Vecchi MP. Optimization by simulated annealing. Science 1983;202:671-80.

[6] McCollum B. International Timetabling Competition 2007. Available at $\langle$ http:// www.cs.qub.ac.uk/itc2007/>; 2013 [accessed December 2012].

[7] Müller T. ITC2007 solver description: a hybrid approach. Ann Oper Res 2009; $172: 429-46$.

[8] University of Twente. International Timetabling Competition 2012. Available at 〈http://www.utwente.nl/ctit/hstt/itc2011/welcome/); 2012 [accessed December 2012].

[9] Fonseca G, Santos H, Toffolo T, Brito S, Souza M. GOAL solver: a hybrid local search based solver for high school timetabling. Ann Oper Res 2014 [in press].

[10] Santos HG, Uchoa E, Ochi LS, Maculan N. Strong bounds with cut and column generation for class-teacher timetabling. Ann Oper Res 2012;194:399-412.

[11] Daskalaki S, Birbas T, Housos E. An integer programming formulation for a case study in university timetabling. Eur J Oper Res 2004;153(1):117-35.

[12] Tripathy A. School timetabling-a case in large binary integer linear programming. Manag Sci 1984;30(12):1473-89.

[13] Haspeslagh S, De Causmaecker P, Stolevik M, Schaerf A. First international nurse rostering competition 2010. Technical report, CODeS. Belgium: Department of Computer Science, KULeuven Campus Kortrijk; 2010.

[14] Post G, Ahmadi S, Daskalaki S, Kyngas J, Nurmi C, Ranson D, et al. An XML format for Benchmarks in High School Timetabling. In: PATAT'08 Proceedings of the 7th international conference on the practice and theory of automated timetabling, vol. 03018, 2008.

[15] Post, Gerhard, Kingston, Jeffrey H, Ahmadi, Samad, Daskalaki, Sophia, Gogos, Christos, Kyngas, Jari, Nurmi, Cimmo, Musliu, Nysret, Pillay, Nelishia, Santos, Haroldo, Schaerf, Andrea. XHSTT: an XML archive for high school timetabling problems in different countries. Ann. Oper. Res. 2011;1-7. <http://dx.doi.org/ 10.1007/s10479-011-1012-2>.

[16] Kingston JH. A tiling algorithm for high school timetabling. Lecture notes in computer science: V practice and theory of automated timetabling, vol. 3616. Berlin: Springer; 2005. p. 208-25.

[17] Wright M. School timetabling using heuristic search. J Oper Res Soc 1996;47: 347-357.

[18] Nurmi K, Kyngas J. A framework for school timetabling problem. In: Proceedings of the 3rd multidisciplinary international scheduling conference: theory and applications, Paris, 2007. p. 386-93.

[19] Valourix C, Housos E. Constraint programming approach for school timetabling. Comput Oper Res 2003;30:1555-72.

[20] de Haan P, Landman R, Post G, Ruizenaar H. A case study for timetabling in Dutch secondary school. Lecture notes in computer science: VI practice and theory of automated timetabling, vol. 3867. Berlin: Springer; 2007. p. 267-79.

[21] Post, Gerhard, Ahmadi, Samad, Daskalaki, Sophia, Kingston, Jeffrey H, Kyngas, Jari, Nurmi, Cimmo, Ranson, David. An XML format for benchmarks in high school Timetabling. Ann. Oper. Res. 2012;194(1):385-397. <http://dx.doi.org/10.1007/ s10479-010-0699-9).

[22] Kingston JH. A software library for school timetabling. Available at $\langle$ http:/ sydney.edu.au/engineering/it/ jeff/khe/ $/$; May 2012.

[23] Kingston JH. Hierarchical timetable construction. In: Problems, proceedings of the first international conference on the practice and theory of automated timetabling, 2006.

[24] Mladenovic N, Hansen P. Variable neighborhood search. Comput Oper Res 1997;24:1097-100.

[25] Costa WE, Goldbarg MC, Goldbarg EFG. New VNS heuristic for total flowtime flowshop scheduling problem. Expert Syst Appl 2012;39:8149-61.

[26] Vlah S, Lukac Z, Pacheco J. Use of VNS heuristics for scheduling of patients in hospital. J Oper Res Soc 2011;62:1227-38.

[27] Wang X, Tang L. A Hybrid VNS with TS for the single machine scheduling problem to minimize the sum of weighted tardiness of jobs. In: Proceedings of the 4th international conference on intelligent computing: advanced intelligent computing theories and applications - with aspects of artificial intelligence. Berlin, Heidelberg: Springer-Verlag; 2008. p. 727-33.

[28] Hansen P, Mladenović N. Variable neighborhood search: principles and applications. Eur J Oper Res 2001;130:449-67.

[29] Hansen P, Mladenović N. Variable neighborhood search: a chapter of handbook of applied optimization. Les Cahiers du GERAD G-2000-3. Montreal, Canada, 2000 (Chapter 8).

[30] Kingston JH. A software library for school timetabling. Available at 〈http:// sydney.edu.au/engineering/it/ jeff/khe/ $/ ; 2012$ [accessed December 2012].

[31] Brito SS, Fonseca GH, Toffolo TA, Santos HG, Souza MJ. A SA-VNS approach for the high school timetabling problem. Electron Notes Discr Math 2012;39: 169-176.

[32] Kochetov Y, Kononova P, Paschenko M. Formulation space search approach for the teacher/class timetabling problem. Yugosl J Oper Res 2008;18(1):1-11. 